\title{
REVIEW
}

\section{The genetic regulation of skeletal muscle development: insights from chicken studies}

\author{
Wen LUO ${ }^{1,2}$, Bahareldin A. ABDALLA ${ }^{1,2}$, Qinghua NIE ${ }^{1,2}$, Xiquan ZHANG $(\bowtie)^{1,2}$ \\ 1 Department of Animal Genetics, Breeding and Reproduction, College of Animal Science, South China Agricultural University, \\ Guangzhou 510642, China \\ 2 Guangdong Provincial Key Lab of Agro-Animal Genomics and Molecular Breeding, and Key Lab of Chicken Genetics, Breeding and \\ Reproduction, Ministry of Agriculture, South China Agricultural University, Guangzhou 510642, China
}

\begin{abstract}
Skeletal muscle development is a complex multi-process trait regulated by various genetic factors. The chicken embryo is an ideal model system for studying skeletal muscle development. However, only a small proportion of the genetic factors affecting skeletal muscle development have been identified in chicken. The aim of this review is to summarize recent knowledge about the genetic factors involved in the regulation of skeletal muscle development in the chicken, such as gene polymorphisms, epigenetic modification, noncoding RNAs and transcription factors, which can influence skeletal muscle development at the genome, epigenome, transcriptome and proteome levels. Research on the regulation of skeletal muscle development in chicken is not yet comprehensive and most of the candidate genes and single nucleotide polymorphisms related to chicken muscle growth remain to be verified in experimental studies. In addition, the data derived from transcriptome sequencing and genome-wide association studies still require further investigation and analysis and comprehensive studies on the regulation of chicken skeletal muscle development will continue as a major research focus.
\end{abstract}

Keywords chicken, epigenetic modification, miRNAs, skeletal muscle development, SNP, transcription factor

\section{Introduction}

Skeletal muscle is a form of striated muscle tissue, distributed mainly in the limbs, chest and hips of vertebrates. Most skeletal muscles are attached to bones by tendons, and are important for movement, strength,

Received January 4, 2017; accepted February 22, 2017

Correspondence: xqzhang@scau.edu.cn metabolism and body posture of the animal. The skeletal muscle is made up of thousands of muscle fibers, each containing multiple nuclei, which come from the fusion of myoblasts. Skeletal muscle development is a complex trait, influenced by genetic regulation and environmental cues $^{[1,2]}$. The developmental process from muscle precursor cell to muscle fiber formation is regulated by various genetic factors, including gene polymorphism, transcription factors, DNA methylation and noncoding RNAs $^{[3-7]}$. These genetic factors cooperate with each other to ensure the normal development of skeletal muscle.

Skeletal muscle development is a multi-step process that includes myofiber formation and hypertrophy. The formation of myofiber occurs mainly in embryogenesis. During the embryonic stage, the myofibers are generated by the following four major processes: myogenic precursor differentiation from somites, myoblast development from myogenic precursors, myoblast proliferation and fusion to form the multinucleated myotubes, and finally maturation of myotubes into myofibers ${ }^{[3,8]}$. After myofibers are formed, they undergo hypertrophy at the postnatal stage. This stage comprises protein turnover, satellite cell proliferation, differentiation and fusion with myofibers to stimulated the hypertrophy of myofibers ${ }^{[9]}$. In addition to these complex cell developmental processes during myofiber formation and hypertrophy, the fine-tuned regulation of numerous myogenic genes is also important for the development of skeletal muscle.

Members of three families of transcription factors have important roles during skeletal muscle development. The paired box proteins, Pax-3 and Pax-7, are essential for muscle precursor cell proliferation, myoblast determination and the specification of myogenic satellite cell ${ }^{[10-14]}$. The myogenic regulatory factor family includes MyoD, myogenin, myogenic factor 5 and myogenic regulatory factor 4 , which are muscle-specific transcription factors that are indispensable for myoblast determination and 
differentiation ${ }^{[15-17]}$. The myocyte enhancer factor 2 family of transcription factors includes MEF2A, -B, -C and-D, which function mainly in myoblast differentiation and muscle-specific gene transcription ${ }^{[18-20]}$. These three transcription factor families cooperate with each other to facilitate myogenesis. In addition to the three transcription factor families, many other genetic factors can also regulate skeletal muscle development. DNA polymorphism in these muscle development-related genes can result in abnormal muscle growth ${ }^{[7,21]}$. MicroRNAs (miRNAs) have been found to have an important regulatory role during skeletal muscle development ${ }^{[3,22]}$. The long non-coding RNAs and the circular RNAs also regulate myogenesis through their own regulatory mechanisms ${ }^{[23,24]}$. Epigenetic modification, such as histone modifications and DNA methylation, can also control skeletal muscle development by epigenetic mechanisms ${ }^{[5,6,25]}$. Studies on all of the above genetic factors have become well established in research on myogenesis regulation. However, the precise regulatory mechanisms and networks involved in skeletal muscle development still need to be elucidated and more and more genetic factors involved in skeletal muscle development remain to be explored.

Myogenic proteins are highly conserved among mammalian and avian embryos ${ }^{[26]}$ and many major discoveries in muscle development depend on avian model systems, especially the chick ${ }^{[27]}$. The chicken is the first model organism to be used and is excellent for developmental investigations ${ }^{[28,29]}$. Additionally, the skeletal muscle of chicken is an important food source for humans. However, the genetic regulation of chicken skeletal muscle development is still poorly understood. In this review, the genetic factors regulating the programs of chicken skeletal muscle development are summarized, and the recent progress in the investigation of chicken myogenic-related regulatory factors newly identified in our laboratory are discussed.

\section{DNA polymorphisms affecting chicken skeletal muscle development}

DNA polymorphisms are a useful tool for finding genetic markers related to chicken phenotypic traits. In this section, single nucleotide polymorphisms (SNPs) that related to chicken skeletal muscle development are summarized and discussed. Most of these SNPs are detected by GWAS, direct genome sequencing and/or SNP genotyping technologies. Since growth traits can to some extent also explain or reflect the status of skeletal muscle development, and skeletal muscles that attached to the trunk, wings and hips have been used by researchers for estimating bodyweight, in this part we also discussed the SNPs that are significantly associated with several production traits related to chicken bodyweight.
2.1 Single nucleotide polymorphisms in the myogenic genes related to chicken skeletal muscle performance

Many genes, called myogenic genes, from three families of transcription factors, myostatin $(M S T N)$ and growth axis related genes have been found to be involved in skeletal muscle development. These myogenic genes are ideal candidate genes for genetic marker screening for broiler breeding. Many polymorphisms have been found to be significantly associated with skeletal muscle growth traits through the screening of genetic markers of myogenic genes (Table 1). These SNPs can be located in the 5'UTR, intron, exon and $3^{\prime} \mathrm{UTR}$ region of the gene. SNPs in the $5^{\prime}$ UTR or intron may influence gene expression, or may be in linkage with some other causative polymorphisms that influences skeletal muscle growth and development ${ }^{[36]}$. In addition, a synonymous mutation, which does not cause an amino acid change, can also affect gene function and muscle development ${ }^{[44]}$. SNPs at the MSTN gene promoter might result in the downregulation of this gene, which is a negative regulator of skeletal muscle development ${ }^{[42]}$. However, the mechanism whereby these SNPs affect chicken skeletal muscle development remain unclear. The major task for the future is to find the relationship between genotype and phenotype. Further exploration of the molecular mechanism underling this relationship is still needed.

2.2 Genome-wide association studies reveal single nucleotide polymorphisms significantly related to chicken muscle growth traits

Skeletal muscle development is a complex trait that can be regulated by multiple genetic factors. As a useful method for discovering complex trait-related gene loci or genomic regions, genome-wide association study (GWAS) has yielded more reproducible associations than many other approaches ${ }^{[49]}$. Five SNPs have been found to be significantly associated with the chicken muscle growth trait in the $1.5 \mathrm{Mb}$ karyopherin subunit alpha 3- forkhead box $\mathrm{O} 1$ a (KPNA3-FOXO1A) region at chicken chromosome 1 (GGA1), which were detected by using GWAS in a chicken $F_{2}$ resource population (White Recessive Rock $\times$ Xinghua ${ }^{[50]}$. One of the 5 SNPs, rs 15497910 , is significantly associated with breast and leg muscle weight, indicating its potential role in the regulation of skeletal muscle development. However, no studies have reported indicating that the KPNA3 gene, which is the nearest gene to rs15497910, is able to influence muscle development in any species. So rs15497910 might influence chicken muscle growth trait by another mechanism that is independent of the function of KPNA3 gene. The other two SNPs, rs13973515 and GGaluGA055359, were significantly associated with average daily weight gain at 15 to $28 \mathrm{~d}$ and wing weight, respectively. In addition, the nearest gene to these two SNPs is FOXO1A, which is an 
Table 1 Single nucleotide polymorphisms located in myogenic genes associated with chicken skeletal muscle development

\begin{tabular}{|c|c|c|c|}
\hline Polymorphism & Gene ID & Skeletal muscle development related trait & Reference \\
\hline$\overline{A 17299834 G}$ & $I G F 1 R$ & Eviscerated weight & [30] \\
\hline G729T & $I G F B P 2$ & Breast muscle weight & [31] \\
\hline A663T & $I G F B P 2$ & Breast muscle weight & \\
\hline G738A & $I G F B P 2$ & Breast muscle weight and leg muscle weight & \\
\hline $\mathrm{C} / \mathrm{T} \mathrm{SNP}$ in intron 2 & $I G F B P 2$ & Bodyweights & [32] \\
\hline g. $570 \mathrm{C}>\mathrm{A}$ & $I G F 1$ & Breast muscle weight & [33] \\
\hline C51978309T & $I G F 1$ & Transversal area of the leg and breast muscle fiber & [34] \\
\hline G6631778A & GHR & Bodyweights & [35] \\
\hline $\mathrm{G}+1705 \mathrm{~A}$ & $G H$ & Bodyweights & [36] \\
\hline MR2 & PIT1 & Bodyweight at $28 \mathrm{~d}, 42 \mathrm{~d}$ & [37] \\
\hline MR4 & PIT1 & Bodyweight at $84 \mathrm{~d}$ & \\
\hline MR5 & PIT1 & Bodyweight at $21 \mathrm{~d}, 28 \mathrm{~d}$ & \\
\hline g. $3051 \mathrm{C}>\mathrm{T}$ & GHSR & Final bodyweight & [38] \\
\hline c. $739+726 \mathrm{~T}>\mathrm{C}(\mathrm{M} 2)$ & GHSR & Breast muscle weight and leg muscle weight & [39] \\
\hline G1215A & GHRL & Bodyweight & [40] \\
\hline $8 b p$ indel & GHRL & Bodyweight & \\
\hline C71T & GHRL & Bodyweight & [41] \\
\hline A241T & MSTN & Bodyweight at hatching & [42] \\
\hline c. $234 \mathrm{G}>\mathrm{A}$ & MSTN & Bodyweights & [43] \\
\hline G2283A & $M S T N$ & Bodyweights & [44] \\
\hline $\mathrm{T} 46023 \mathrm{C}$ & $M E F 2 A$ & Leg muscle weight & [45] \\
\hline $\mathrm{T} 89232 \mathrm{G}$ & $M E F 2 A$ & Semi-eviscerated percentage & \\
\hline $87 \mathrm{~T}>\mathrm{C}$ & Myf5 & Eviscerated weight & [46] \\
\hline $154 \mathrm{~T}>\mathrm{C}$ & $M S T N$ & Breast muscle weight & \\
\hline A446G & MSTN & Breast muscle percentage & [47] \\
\hline 31-bp indel & PAX7 & Breast muscle fiber diameter, leg muscle fiber diameter, breast muscle fiber density & [48] \\
\hline
\end{tabular}

important transcription factor during skeletal muscle development ${ }^{[51-53]}$. The question whether and how these two SNPs can influence expression or function of FOXO1A needs further study.

Two other GWAS of a chicken $\mathrm{F}_{2}$ resource population derived from the reciprocal crosses between slow- and fastgrowing chicken breeds, have also identified some loci or genes related to muscle growth ${ }^{[54,55]}$. Unlike the results described above, these two studies showed that many SNPs on GGA4 are significantly associated with chicken growth traits. The genes near significant SNPs, such as ligand dependent nuclear receptor corepressor-like protein 1, LIM domain binding 2 and microtubule-associated protein tau, have lower expression in breast muscle of slow-growing chickens compared to fast-growing chickens, indicating their potential roles in the regulation of muscle development ${ }^{[54]}$. By GWAS of local chicken breeds, a $0.65 \mathrm{Mb}$ region on GGA3 was identified as associated with breast muscle weight $(\mathrm{BrW})$ and breast muscle percentage $(\mathrm{BrP})^{[56]}$. The gap junction protein alpha 1 (GJA1) gene which is located in this region may be a functional gene for skeletal muscle development, because the expression of this gene was upregulated with the increase of breast muscle weight across development ${ }^{[56]}$. Additionally, four SNPs (GGaluGA225255, Gga rs16287013, Gga_rs14366866 and Gga_rs14366948) located near GJA1 are significantly associated with BrW and BrP. Another GWAS, using Jinghai yellow chickens, found that 15 SNPs are significantly associated with five carcass traits $^{[57]}$. However, there has been no SNP identified by GWAS that is significantly associated with breast muscle weight or leg muscle weight, which are more representative of skeletal muscle development.

Even though increasing numbers of SNPs, genes and regions have been found to be associated with chicken skeletal muscle development by GWAS, none of the new candidate genes has been verified to be involved in skeletal muscle development. The significant associated loci identified in the genome by these studies are very different, indicating that the loci associated with muscle 
development are not consistent between breeds. Thus GWAS cannot fully exploit all loci associated with muscle development and a more comprehensive and deeper analysis of these GWAS results is still needed.

\section{Transcriptional level regulation of chicken skeletal muscle development}

3.1 Identification of candidate genes involved in chicken skeletal muscle development by microarray and highthroughput sequencing

Gene expression profiling chips and high-throughput sequencing are effective methods for screening differentially expressed genes between groups. By using skeletal muscle from slow- and fast-growing chicken breeds as experimental materials, or using skeletal muscle from different developmental stages of chicken, researchers have found many candidate genes related to skeletal muscle development using microarray or high-throughput sequencing experiments. By sequencing RNA to detect gene expression difference between the skeletal muscle of recessive white rock (WRR) and Xinghua (XH) chicken, higher expression of $\mathrm{FOXO} 3$ was found to potentially inhibit skeletal muscle development of XH chicken ${ }^{[58]}$. This could be because the mRNA level of FOXO3 is expressed more highly in the skeletal muscle of $\mathrm{XH}$ than that in WRR chickens, and reduced $F O X O 3$ expression can upregulate growth-related genes expression in DF-1 cells $^{[58]}$. In addition to WRR and XH chickens, skeletal muscle from sex-linked dwarf (SLD) chicken and normal chicken have been used to discover genes or pathways related to chicken muscle development ${ }^{[59,60]}$. The SLD chicken has lower bodyweight and smaller muscle fibers than those of normal chicken because of a recessive mutation of the growth hormone receptor $(G H R)$ gene. The GHR mutation leads 55 genes and 173 genes to be differentially expressed between the two chicken breeds in embryonic day 14 (E14) and week 7 (W7), respectively, and these genes are mainly related to the regulation of cell division ${ }^{[59]}$. By comparing the differentially expressed genes between E14 and W7 in SLD and normal chickens, it was also found that many genes enriched in the MAPK, PI3K-Akt, Wnt and insulin pathways were related to chicken skeletal muscle development, and these genes can interact with miRNAs to construct a miRNAs-mRNAs regulatory network during skeletal muscle development ${ }^{[59]}$. The above study was performed using microarray experiments to investigate gene expression. Similarly, microarrays were used to study Qingyuan partridge and Cobb 500, representing slow- and fast-growing chicken breeds, respectively ${ }^{[61]}$. In that study, many differentiallyexpressed genes were also found, and it was argued that energy metabolism-related genes might influence muscle development, such as protein kinase AMP-activated noncatalytic subunit gamma 1, protein kinase AMP-activated non-catalytic subunit gamma 3 and protein kinase cAMPdependent type II regulatory subunit beta ${ }^{[61]}$.

Broilers and layers are normally different chicken breeds that differ enormously in their growth rates and body sizes. By comparing gene expression in the skeletal muscle of these two types of chicken, researchers have found that many genes related to muscle fiber types, satellite cell proliferation and differentiation are different in the two kinds of chicken ${ }^{[62]}$. The dickkopf homolog 3 gene could be a potential regulator of skeletal muscle development, because the expression pattern of this gene was significantly correlated with the natural increased rate of body mass of chickens, and it had higher expression level in broilers than in layers ${ }^{[62]}$. In general, although many potential candidate genes were found to be involved in chicken skeletal muscle development, large-scale analyses are still required to validate their roles during skeletal muscle development, to confirm that a candidate gene that can directly affect chicken skeletal muscle development is important for application in broiler breeding.

\subsection{MiRNAs and long non-coding RNAs involved in chicken skeletal muscle development}

MiRNAs regulate target gene expression at the posttranscription level. During skeletal muscle development, many miRNAs have been found to have regulatory roles at each step ${ }^{[3,22]}$. However, most of this research has been in mammals, and the question whether these miRNAs can regulate chicken skeletal muscle development still remains to be elucidated. To investigate the functional miRNAs in chicken skeletal muscle development, miRNAs and mRNAs were sequenced from the skeletal muscle of both broilers and layers ${ }^{[62]}$. A miRNA-target gene interaction network related to skeletal muscle development was constructed by using integrative miRNA targetprediction and network analysis. In that network, many genes related to muscle development were found to be targeted by miRNAs, for example, gga-miR-1a can directly inhibit activin A receptor type $2 \mathrm{~B}$ expression, and reversion inducing cysteine rich protein with kazal motifs is a putative target of gga-miR-200b ${ }^{[62]}$. A similar research strategy has been used in the study of miRNA deep sequencing in the skeletal muscle of WRR and XH chickens, and to construct a miRNAs-mRNAs interaction network for chicken muscle development ${ }^{[63]}$. However, the detailed functional validation of the genes or miRNAs related to muscle development is still lacking in both of these two studies.

In other work, expression of miRNAs and mRNAs was detected in the skeletal muscle of dwarf and normal 
chickens, and it was found that let- $7 \mathrm{~b}$ can regulate skeletal muscle development by directly inhibiting the GHR gene $^{[60]}$. Even though detailed functional verification of let-7b was not performed, many other candidate miRNAs were found from the microarray results, such as miR-203, miR-20a-5p and miR-20b-5p. By using in vivo detection and in vitro experiments, miR-203 was confirmed to inhibit chicken myoblast proliferation and differentiation by repressing Jun proto-oncogene $(c-J U N)$ and $M E F 2 C$, respectively ${ }^{[64]}$. Similarly, both miR-20a-5p and miR$20 \mathrm{~b}-5 \mathrm{p}$ have been comprehensively validated as regulating chicken myoblast proliferation and differentiation through specifically interacting with E2F transcription factor 1 $(E 2 F 1)^{[65]}$. This work has revealed the regulatory mechanisms of candidate miRNAs during muscle development and also furthered understanding of the regulatory networks of chicken skeletal muscle development.

The candidate gene validation approach is a useful strategy to find a functional miRNA acting during chicken skeletal muscle development. As a well-known myogenic miRNA in mammals, miR-206 has important roles during muscle development. However, its function in chicken still remains unknown. Aiming to investigate miR-206 roles in chicken, miR-206 was overexpressed in chicken myoblasts and found to significantly increase myogenin and muscle creatine kinase expression, which are important genes for muscle differentiation ${ }^{[66]}$. Additionally, eight variants have been identified in the promoter region of $m i R-206$ gene that exhibit significant effects on chicken birthweight, indicating an important role of miR-206 in chicken development. Myomaker is a new gene that was found to be involved in muscle development ${ }^{[67]}$. To find out which miRNA can directly bind to Myomaker 3'UTR and regulate its expression, RNAhybrid software and dual-luciferase reporter assay were used. It was found that miR-140-3p directly inhibited Myomaker expression and by targeting the Myomaker gene inhibits chicken myoblast fusion.

In addition to miRNAs, long non-coding RNAs (lncRNAs) have attracted increasing attention in the study of skeletal muscle development. The first chicken lncRNA catalog in skeletal muscle has been constructed $^{[68]}$. However, although novel lncRNAs in chicken were identified, no single lncRNA has been validated to function during chicken skeletal muscle development. Recently, differences in lncRNAs expression were detected in the skeletal muscle between slow- and fast-growing chicken breeds, and it was found some lncRNAs can regulate myoblast proliferation and differentiation by interacting with miRNAs and mRNAs ${ }^{[69]}$. Additionally, some lncRNAs have also been found to regulate gene expression by cis-regulation (unpublished data). Besides IncRNA, the functions of some circular RNAs during chicken skeletal muscle development have been investigated using circle-RNA sequencing. This research has provided further understanding of the regulation of chicken skeletal muscle development.

\section{Epigenetic modification regulates chicken skeletal muscle growth}

Epigenetics is the study of stably heritable traits or gene expression caused by mechanisms other than underlying DNA sequence changes ${ }^{[70]}$. During skeletal muscle development, epigenetic regulators are able to promote the transcription of a selective group of gene and drive myogenesis ${ }^{[5]}$. Gene expression during myogenesis can also be regulated by epigenetic modification, such as DNA methylation. To identify candidate genes and genomic methylated regions for chicken skeletal muscle development, the genome-wide DNA methylation pattern of skeletal muscle was investigated using methylated DNA immunoprecipitation-sequencing in high and low bodyweights of 7-week-old WRR and XH chickens ${ }^{[71]}$. Many well-known growth-related genes, such as insulin like growth factor 1 receptor $(I G F 1 R)$, fibroblast growth factor 12 (FGF12), FGF14 and fibroblast growth factor receptor 2, were found to exhibit altered DNA methylation in all comparisons, indicating that DNA methylation in several growth-related genes may affect chicken skeletal muscle development.

Another study investigating DNA methylation status in chicken skeletal muscle was conducted in a three-yellow chicken population ${ }^{[72]}$. By using fluorescence-labeled methylation-sensitive amplified polymorphism analysis, it was found that the differences in DNA methylation levels are significantly associated with muscle fiber density and muscle drip loss ${ }^{[72]}$. The level of DNA methylation in the majority of the genome changes dramatically during early development ${ }^{[73]}$. Using broilers as experimental animals, the genomic DNA methylation status was examined during chicken embryogenesis. An increasing genomic DNA methylation level was found in muscle, while the methylation level of the $I G F 2$ promoter gradually decreased $^{[74]}$. As IGF2 is a positive regulator of skeletal muscle development, and DNA methylation in gene promoters can inhibit gene transcription and expression $^{[75]}$, the decreased promoter methylation levels of $I G F 2$ could release the expression of $I G F 2$, and therefore promote skeletal muscle development.

\section{Transcription factor regulates chicken skeletal muscle development through inter- action with miRNAs}

The critical roles of the three transcription factor families during skeletal muscle development have been introduced above. Undoubtedly these transcription factors also have roles in chickens, and the polymorphisms in these factors are associated with chicken muscle growth traits (Table 1). In chicken myoblasts, it was found that MyoD and 
myogenin can promote the transcription of muscle-specific genes, such as Myomaker, by directly binding to the E-box region located in the gene promoter ${ }^{[76]}$. In addition to regulating the transcription of their downstream genes, these transcription factors can also interact with miRNAs and then influence chicken muscle development. Recently, a miRNA that exhibits gradually upregulated expression during chicken myoblast differentiation was found to be regulated by MyoD because of the binding of MyoD to the promoter of the miRNA gene (unpublished data). The MyoD promotes the expression of this miRNA and facilities myoblast differentiation. In other work, it was found that a small RNA called miR-203 can inhibit the expression of $M E F 2 C$, a member of the three transcription factors families, by directly binding to its $3^{\prime} \mathrm{UTR}^{[64]}$. The inhibition of $M E F 2 C$ by miR-203 repressed chicken myoblast differentiation, and reduced the formation of myotubes $^{[64]}$.

In addition to the members of these three transcription factor families, many other transcription factors also have important roles in the regulation of chicken skeletal muscle development (Fig. 1). As important regulators of cell proliferation, c-JUN and E2F1 promote chicken myoblast proliferation by the regulation of their downstream target genes $^{[64,65]}$. Similarly, both can be regulated by miRNA. MiR-203 and miR-20a-5p/20b-5p directly inhibit c-JUN and $E 2 F 1$ expression, respectively, and therefore repress chicken myoblast proliferation ${ }^{[64,65]}$. However, E2F1 can in turn directly regulate the transcription of miR-20a-5p/ $20 b-5 p$ and thus form an E2F1-miR-20a-5p/20b-5p autoregulatory feedback loop ${ }^{[65]}$. In addition to $c-J U N$ and $M E F 2 C$, miR-203 can also bind to the 3'UTR of tumor protein $\mathrm{p} 63$ (p63), which is able to promote muscle cell proliferation and differentiation ${ }^{[77,78]}$. The binding of miR203 to the $p 63$ 3'UTR inhibits its mRNA expression and functions ${ }^{[64]}$. Recently, it was observed that $\mathrm{v}$-myc avian myelocytomatosis viral oncogene homolog (c-Myc) transcription factor can bind to the promoters of a large number of genes, miRNAs and lincRNAs in chicken muscle cells (unpublished data). In vitro experiments showed that cMyc regulates muscle cell proliferation and differentiation by controlling the transcription of these downstream genes, miRNAs and long intergenic non-coding RNAs (lincRNAs). Notably, some of c-Myc target miRNAs can in turn bind to the 3'UTR of $c-M y c$ mRNA and inhibit $c-M y c$ expression. Therefore, the interaction of $\mathrm{c}-\mathrm{Myc}$ and its downstream miRNAs provide a potentially important feedback loop during chicken skeletal muscle development.

\section{Conclusions and future perspectives}

Chicken embryos are a perfect model system for research

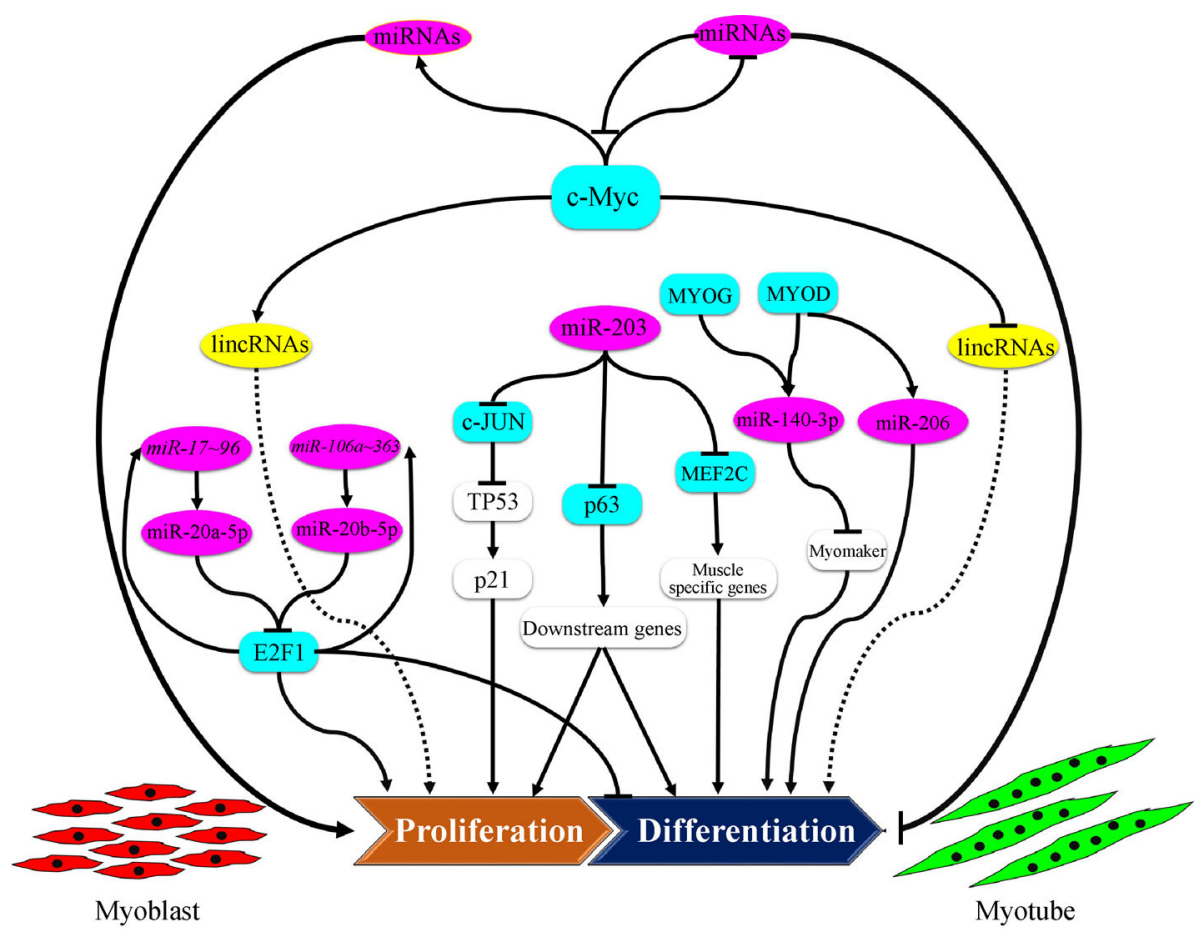

Fig. 1 The network of transcription factors and miRNAs interacting during chicken myoblasts differentiation to myotubes. Light blue represents transcription factors; red represents miRNAs; yellow represents lincRNAs; white represents genes. 
on skeletal muscle development. Studies on the regulation of chicken skeletal muscle development not only provide further understanding of the process of muscle fiber formation, but also allow the identification of potential candidate genes and molecular markers that could improve chicken muscle mass. In recent years, many genes, SNPs, DNA methylation regions and noncoding RNAs have been found to be involved in chicken skeletal muscle development, especially during the processes of myoblast proliferation and differentiation. These two processes are important for the formation of muscle fiber and determination of muscle fiber number. However, only a few genetic regulators involved in the control of these two processes were found in chicken, including some miRNAs and transcription factors. Dozens of genes, ncRNAs and epigenetic modification involved in the regulation of chicken skeletal muscle development still need to be validated.

The regulatory function of DNA methylation of gene promoters, IncRNAs and circRNAs should be the next area of research focus for chicken muscle development. Also, the interaction between transcription factors and noncoding RNAs should be a priority for further study. Additionally, the reduced costs of sequencing and microarray have enabled the wider use of GWAS for identifying complex trait-related gene loci and genomic regions. Unlike humans and mice, the functions of most of the candidate genes or loci identified by GWAS in chickens are still to be validated empirically. Therefore, experimental validation of function of the candidate genes and loci involved in chicken skeletal muscle development should also be a priority. In addition, gene imprinting, histone acetylation, protein modification and protein structural polymorphism have gradually been attracting more attention in cell development research ${ }^{[79]}$. The way in which these genetic factors regulate skeletal muscle development should also receive more attention. Finally, the rate of skeletal muscle development is usually associated with meat quality in the production of livestock and poultry. However, the challenge is to improve the growth rate of skeletal muscle while in parallel ensuring the meat quality. To achieve this the characteristics of skeletal muscle during the process of development that affect meat quality must be understood. All of these issues deserve further study.

Acknowledgements This work was supported by the National Natural Science Foundation of China (31172200), the China Agriculture Research System (CARS-42-G05), the Program for New Century Excellent Talents in University (NCET-13-0803), and the Foundation for High-level Talents in Higher Education of Guangdong, China.

Compliance with ethics guidelines Wen Luo, Bahareldin A. Abdalla, Qinghua Nie, and Xiquan Zhang declare that they have no conflicts of interest or financial conflicts to disclose.

This article is a review and does not contain any studies with human or animal subjects performed by any of the authors.

\section{References}

1. Ge Y, Sun Y, Chen J. IGF-II is regulated by microRNA-125b in skeletal myogenesis. Journal of Cell Biology, 2011, 192(1): 69-81

2. Perry R L, Rudnick M A. Molecular mechanisms regulating myogenic determination and differentiation. Frontiers in Bioscience-landmark, 2000, 5(3): D750-D767

3. Luo W, Nie Q, Zhang X. MicroRNAs involved in skeletal muscle differentiation. Journal of Genetics and Genomics, 2013, 40(3): 107-116

4. Berkes C A, Tapscott S J. MyoD and the transcriptional control of myogenesis. Seminars in Cell \& Developmental Biology, 2005, 16 (4-5): 585-595

5. Saccone V, Puri P L. Epigenetic regulation of skeletal myogenesis. Organogenesis, 2010, 6(1): 48-53

6. Palacios D, Puri P L. The epigenetic network regulating muscle development and regeneration. Journal of Cellular Physiology, 2006, 207(1): 1-11

7. Clop A, Marcq F, Takeda H, Pirottin D, Tordoir X, Bibé B, Bouix J, Caiment F, Elsen J M, Eychenne F, Larzul C, Laville E, Meish F, Milenkovic D, Tobin J, Charlier C, Georges M. A mutation creating a potential illegitimate microRNA target site in the myostatin gene affects muscularity in sheep. Nature Genetics, 2006, 38(7): 813-818

8. Feng Y, Cao J H, Li X Y, Zhao S H. Inhibition of miR-214 expression represses proliferation and differentiation of $\mathrm{C} 2 \mathrm{C} 12$ myoblasts. Cell Biochemistry and Function, 2011, 29(5): 378-383

9. Oksbjerg N, Gondret F, Vestergaard M. Basic principles of muscle development and growth in meat-producing mammals as affected by the insulin-like growth factor (IGF) system. Domestic Animal Endocrinology, 2004, 27(3): 219-240

10. Seale P, Sabourin L A, Girgis-Gabardo A, Mansouri A, Gruss P, Rudnicki M A. Pax7 is required for the specification of myogenic satellite cells. Cell, 2000, 102(6): 777-786

11. Relaix F, Montarras D, Zaffran S, Gayraud-Morel B, Rocancourt D, Tajbakhsh S, Mansouri A, Cumano A, Buckingham M. Pax3 and Pax7 have distinct and overlapping functions in adult muscle progenitor cells. Journal of Cell Biology, 2006, 172(1): 91-102

12. Dulak J. Many roles for Pax7. Cell Cycle, 2017, 16(1): 21-22

13. Finckenstein F G, Davicioni E, Osborn K G, Cavenee W K, Arden K C, Anderson M J. Transgenic mice expressing PAX3-FKHR have multiple defects in muscle development, including ectopic skeletal myogenesis in the developing neural tube. Transgenic Research, 2006, 15(5): 595-614

14. Buckingham M, Relaix F. PAX3 and PAX7 as upstream regulators of myogenesis. Seminars in Cell \& Developmental Biology, 2015, 44: $115-125$

15. Rudnicki M A, Jaenisch R. The MyoD family of transcription factors and skeletal myogenesis. BioEssays, 1995, 17(3): 203-209

16. Megeney L A, Rudnicki M A. Determination versus differentiation and the MyoD family of transcription factors. Biochemistry and Cell Biology-Biochimie Et Biologie Cellulaire, 1995, 73(9-10): 723-732

17. Wood W M, Etemad S, Yamamoto M, Goldhamer D J. MyoDexpressing progenitors are essential for skeletal myogenesis and satellite cell development. Developmental Biology, 2013, 384(1): $114-127$ 
18. Black B L, Olson E N. Transcriptional control of muscle development by myocyte enhancer factor-2 (MEF2) proteins. Annual Review of Cell and Developmental Biology, 1998, 14(1): 167-196

19. Potthoff M J, Olson E N. MEF2: a central regulator of diverse developmental programs. Development, 2007, 134(23): 4131-4140

20. Estrella N L, Desjardins C A, Nocco S E, Clark A L, Maksimenko $\mathrm{Y}$, Naya F J. MEF2 transcription factors regulate distinct gene programs in mammalian skeletal muscle differentiation. Journal of Biological Chemistry, 2015, 290(2): 1256-1268

21. Grobet L, Martin L J, Poncelet D, Pirottin D, Brouwers B, Riquet J, Schoeberlein A, Dunner S, Menissier F, Massabanda J, Fries R, Hanset R, Georges M.A deletion in the bovine myostatin gene causes the double-muscled phenotype in cattle. Nature Genetics, 1997, 17(1):7 1-74

22. van Rooij E, Liu N, Olson E N. MicroRNAs flex their muscles. Trends in Genetics, 2008, 24(4): 159-166

23. Gong C, Li Z, Ramanujan K, Clay I, Zhang Y, Lemire-Brachat S, Glass D J. A long non-coding RNA, LncMyoD, regulates skeletal muscle differentiation by blocking IMP2-mediated mRNA translation. Developmental Cell, 2015, 34(2): 181-191

24. Cesana M, Cacchiarelli D, Legnini I, Santini T, Sthandier O, Chinappi M, Tramontano A, Bozzoni I. A long noncoding RNA controls muscle differentiation by functioning as a competing endogenous RNA. Cell, 2011, 147(2): 358-369

25. Sousa-Victor P, Muñoz-Cánoves P, Perdiguero E. Regulation of skeletal muscle stem cells through epigenetic mechanisms. Toxicology Mechanisms and Methods, 2011, 21(4): 334-342

26. Mok G F, Sweetman D. Many routes to the same destination: lessons from skeletal muscle development. Reproduction, 2011, 141 (3): $301-312$

27. Hirst C E, Marcelle C. The avian embryo as a model system for skeletal myogenesis. Results and Problems in Cell Differentiation, 2015, 56: 99-122

28. Stern C D. The chick: a great model system becomes even greater. Developmental Cell, 2005, 8(1): 9-17

29. Tickle C. The contribution of chicken embryology to the understanding of vertebrate limb development. Mechanisms of Development, 2004, 121(9): 1019-1029

30. Lei M, Peng X, Zhou M, Luo C, Nie Q, Zhang X. Polymorphisms of the $I G F 1 R$ gene and their genetic effects on chicken early growth and carcass traits. BMC Genetics, 2008, 9(1): 70

31. Lei M M, Nie Q H, Peng X, Zhang D X, Zhang X Q. Single nucleotide polymorphisms of the chicken insulin-like factor binding protein 2 gene associated with chicken growth and carcass traits. Poultry Science, 2005, 84(8): 1191-1198

32. Li Z H, Li H, Zhang H, Wang S Z, Wang Q G, Wang Y X. Identification of a single nucleotide polymorphism of the insulinlike growth factor binding protein 2 gene and its association with growth and body composition traits in the chicken. Journal of Animal Science, 2006, 84(11): 2902-2906

33. Sato S, Ohtake T, Uemoto Y, Okumura Y, Kobayashi E. Polymorphism of insulin-like growth factor 1 gene is associated with breast muscle yields in chickens. Animal Science Journal, 2012, 83(1): 1-6

34. Lei M, Luo C, Peng X, Fang M, Nie Q, Zhang D, Yang G, Zhang X.
Polymorphism of growth-correlated genes associated with fatness and muscle fiber traits in chickens. Poultry Science, 2007, 86(5): 835-842

35. Ouyang J H, Xie L, Nie Q, Luo C, Liang Y, Zeng H, Zhang X. Single nucleotide polymorphism (SNP) at the GHR gene and its associations with chicken growth and fat deposition traits. British Poultry Science, 2008, 49(2): 87-95

36. Nie Q, Sun B, Zhang D, Luo C, Ishag N A, Lei M, Yang G, Zhang $\mathrm{X}$. High diversity of the chicken growth hormone gene and effects on growth and carcass traits. Journal of Heredity, 2005, 96(6): 698703

37. Nie Q, Fang M, Xie L, Zhou M, Liang Z, Luo Z, Wang G, Bi W, Liang $\mathrm{C}$, Zhang $\mathrm{W}$, Zhang $\mathrm{X}$. The PITl gene polymorphisms were associated with chicken growth traits. BMC Genetics, 2008, 9(1): 20

38. Kaczor U, Poltowicz K, Kucharski M, Sitarz A M, Nowak J, Wojtysiak D, Zieba D A.Effect of ghrelin and leptin receptors genes polymorphisms on production results and physicochemical characteristics of M. pectoralis superficialis in broiler chickens. Animal Production Science, 2016, 1(57): 42-50

39. Fang M, Nie Q, Luo C, Zhang D, Zhang X. Associations of GHSR gene polymorphisms with chicken growth and carcass traits. Molecular Biology Reports, 2010, 37(1): 423-428

40. Fang M, Nie Q, Luo C, Zhang D, Zhang X. An 8bp indel in exon 1 of Ghrelin gene associated with chicken growth. Domestic Animal Endocrinology, 2007, 32(3): 216-225

41. Li C C, Li K, Li J, Mo D L, Xu R F, Chen G H, Qiangba Y Z, Ji S L, Tang X H, Fan B, Zhu M J, Xiong T A, Guan X, Liu B. Polymorphism of ghrelin gene in twelve Chinese indigenous chicken breeds and its relationship with chicken growth traits. Asian-Australasian Journal of Animal Sciences, 2006, 19(2): 153159

42. Paswan C, Bhattacharya $\mathrm{T} K$, Nagaraj C S, Chaterjee R N, Jayashankar M R. SNPs in minimal promoter of myostatin (GDF-8) gene and its association with body weight in broiler chicken. Journal of Applied Animal Research, 2014, 42(3): 304-309

43. Zhang G X, Zhao X H, Wang J Y, Ding F X, Zhang L. Effect of an exon 1 mutation in the myostatin gene on the growth traits of the Bian chicken. Animal Genetics, 2012, 43(4): 458-459

44. Zhang G, Ding F, Wang J, Dai G, Xie K, Zhang L, Wang W, Zhou S. Polymorphism in exons of the myostatin gene and its relationship with body weight traits in the Bian chicken. Biochemical Genetics, 2011, 49(1-2): 9-19

45. Zhou Y, Liu Y, Jiang X, Du H, Li X, Zhu Q. Polymorphism of chicken myocyte-specific enhancer-binding factor $2 \mathrm{~A}$ gene and its association with chicken carcass traits. Molecular Biology Reports, 2010, 37(1): 587-594

46. Yin H, Zhang Z, Lan X, Zhao X, Wang Y, Zhu Q. Association of $M y F 5, M y F 6$ and $M y o G$ gene polymorphisms with carcass traits in Chinese Meat Type Quality chicken populations. Journal of Animal and Veterinary Advances, 2011, 10(6): 704-708

47. Liu H, Xu Y, Xiong B, Hu W, Mei H, Shi H, Zuo B, Nie X, Wang H. Study on the Correlation between Polymorphisms of $M y o G$ Gene and Growth Traits in Luning Chicken. China Animal Husbandry \& Veterinary Medicine, 2015, 42(3): 629-637

48. Zhang S, Han R L, Gao Z Y, Zhu S K, Tian Y D, Sun G R, Kang X $\mathrm{T}$. A novel 31-bp indel in the paired box 7 (PAX7) gene is associated 
with chicken performance traits. British Poultry Science, 2014, 55 (1): $31-36$

49. Nicolae D L, Gamazon E, Zhang W, Duan S, Dolan M E, Cox N J. Trait-associated SNPs are more likely to be eQTLs: annotation to enhance discovery from GWAS. PLoS Genetics, 2010, 6(4): e1000888

50. Xie L, Luo C, Zhang C, Zhang R, Tang J, Nie Q, Ma L, Hu X, Li N, Da Y, Zhang X. Genome-wide association study identified a narrow chromosome 1 region associated with chicken growth traits. PLoS One, 2012, 7(2): e30910

51. Kamei Y, Miura S, Suzuki M, Kai Y, Mizukami J, Taniguchi T, Mochida K, Hata T, Matsuda J, Aburatani H, Nishino I, Ezaki O. Skeletal muscle FOXO1 (FKHR) transgenic mice have less skeletal muscle mass, down-regulated Type I (slow twitch/red muscle) fiber genes, and impaired glycemic control. Journal of Biological Chemistry, 2004, 279(39): 41114-41123

52. Kitamura T, Kitamura Y I, Funahashi Y, Shawber C J, Castrillon D H, Kollipara R, DePinho R A, Kitajewski J, Accili D. A Foxo/Notch pathway controls myogenic differentiation and fiber type specification. Journal of Clinical Investigation, 2007, 117(9): 2477-2485

53. Yuan Y, Shi X E, Liu Y G, Yang G S. FoxO1 regulates muscle fibertype specification and inhibits calcineurin signaling during $\mathrm{C} 2 \mathrm{C} 12$ myoblast differentiation. Molecular and Cellular Biochemistry, 2011, 348(1-2): 77-87

54. Liu R, Sun Y, Zhao G, Wang H, Zheng M, Li P, Liu L, Wen J. Identification of loci and genes for growth related traits from a genome-wide association study in a slow- $\times$ fast-growing broiler chicken cross. Genes \& Genomics, 2015, 37(10): 829-836

55. Gu X, Feng C, Ma L, Song C, Wang Y, Da Y, Li H, Chen K, Ye S, $\mathrm{Ge} \mathrm{C}, \mathrm{Hu} \mathrm{X}, \mathrm{Li}$ N. Genome-wide association study of body weight in chicken F2 resource population. PLoS One, 2011, 6(7): e21872

56. Liu R, Sun Y, Zhao G, Wang F, Wu D, Zheng M, Chen J, Zhang L, $\mathrm{Hu}$ Y, Wen J. Genome-wide association study identifies Loci and candidate genes for body composition and meat quality traits in Beijing-You chickens. PLoS One, 2013, 8(4): e61172

57. Wang W, Zhang T, Wang J, Zhang G, Wang Y, Zhang Y, Zhang J, Li G, Xue Q, Han K, Zhao X, Zheng H. Genome-wide association study of 8 carcass traits in Jinghai Yellow chickens using specificlocus amplified fragment sequencing technology. Poultry Science, 2016, 95(3): 500-506

58. Chen B, Xu J, He X, Xu H, Li G, Du H, Nie Q, Zhang X. A genomewide mRNA screen and functional analysis reveal $\mathrm{FOXO3}$ as a candidate gene for chicken growth. PLoS One, 2015, 10(9): e0137087

59. Luo W, Lin S, Li G, Nie Q, Zhang X. Integrative analyses of miRNA-mRNA interactions reveal let-7b, miR-128 and MAPK pathway involvement in muscle mass loss in sex-linked dwarf chickens. International Journal of Molecular Sciences, 2016, 17(3): 276

60. Lin S, Li H, Mu H, Luo W, Li Y, Jia X, Wang S, Jia X, Nie Q, Li Y, Zhang X. Let-7b regulates the expression of the growth hormone receptor gene in deletion-type dwarf chickens. BMC Genomics, 2012, 13(1): 306

61. Du Y F, Ding Q L, Li Y M, Fang W R. Identification of differentially expressed genes and pathways for myofiber characteristics in soleus muscles between chicken breeds differing in meat quality. Animal Biotechnology, 2017, 28 (2): 83-93

62. Zheng Q, Zhang Y, Chen Y, Yang N, Wang X J, Zhu D. Systematic identification of genes involved in divergent skeletal muscle growth rates of broiler and layer chickens. BMC Genomics, 2009, 10(1): 87

63. Ouyang H, He X, Li G, Xu H, Jia X, Nie Q, Zhang X. Deep sequencing analysis of miRNA expression in breast muscle of fastgrowing and slow-growing broilers. International Journal of Molecular Sciences , 2015, 16(7): 16242-16262

64. Luo W, Wu H, Ye Y, Li Z, Hao S, Kong L, Zheng X, Lin S, Nie Q, Zhang X. The transient expression of miR-203 and its inhibiting effects on skeletal muscle cell proliferation and differentiation. Cell Death \& Disease, 2014, 5(7): e1347

65. Luo W, Li G, Yi Z, Nie Q, Zhang X. E2F1-miR-20a-5p/20b-5p auto-regulatory feedback loop involved in myoblast proliferation and differentiation. Scientific Reports, 2016, 6(1): 27904

66. Jia X, Lin H, Abdalla B A, Nie Q. Characterization of miR-206 promoter and its association with birthweight in chicken. International Journal of Molecular Sciences , 2016, 17(4): 559

67. Millay D P, O'Rourke J R, Sutherland L B, Bezprozvannaya S, Shelton J M, Bassel-Duby R, Olson E N. Myomaker is a membrane activator of myoblast fusion and muscle formation. Nature, 2013, 499(7458): 301-305

68. Li T, Wang S, Wu R, Zhou X, Zhu D, Zhang Y. Identification of long non-protein coding RNAs in chicken skeletal muscle using next generation sequencing. Genomics, 2012, 99(5): 292-298

69. Li Z, Ouyang H, Zheng M, Cai B, Han P, Abdalla B A, Nie Q, Zhang X. Integrated analysis of long non-coding RNAs (lncRNAs) and mRNA expression profiles reveals the potential role of lncRNAs in skeletal muscle development of the chicken. Frontiers in Physiology, 2017, 7(7): 687

70. Gottesfeld J M. Introduction to the thematic minireview series on epigenetics. Journal of Biological Chemistry, 2011, 286(21): 18345-18346

71. Hu Y, Xu H, Li Z, Zheng X, Jia X, Nie Q, Zhang X. Comparison of the genome-wide DNA methylation profiles between fast-growing and slow-growing broilers. PLoS One, 2013, 8(2): e56411

72. Zhang Y, Guo J, Gao Y, Niu S, Yang C, Bai C, Yu X, Zhao Z. Genome-wide methylation changes are associated with muscle fiber density and drip loss in male three-yellow chickens. Molecular Biology Reports, 2014, 41(5): 3509-3516

73. Geiman T M, Muegge K. DNA methylation in early development. Molecular Reproduction and Development, 2010, 77(2): 105-113

74. Li S, Zhu Y, Zhi L, Han X, Shen J, Liu Y, Yao J, Yang X. DNA methylation variation trends during the embryonic development of chicken. PLoS One, 2016, 11(7): e0159230

75. Yin H, Blanchard K L. DNA methylation represses the expression of the human erythropoietin gene by two different mechanisms. Blood, 2000, 95(1): 111-119

76. Luo W, Li E, Nie Q, Zhang X. Myomaker, regulated by MYOD, MYOG and miR-140-3p, promotes chicken myoblast fusion. International Journal of Molecular Sciences , 2015, 16(11): 26186-26201

77. Cefalù S, Lena A M, Vojtesek B, Musarò A, Rossi A, Melino G, Candi E. TAp63gamma is required for the late stages of myogenesis. Cell Cycle, 2015, 14(6): 894-901

78. Cam H, Griesmann H, Beitzinger M, Hofmann L, Beinoraviciute- 
Kellner R, Sauer M, Hüttinger-Kirchhof N, Oswald C, Friedl P, Gattenlöhner S, Burek C, Rosenwald A, Stiewe T. p53 family members in myogenic differentiation and rhabdomyosarcoma development. Cancer Cell, 2006, 10(4): 281-293
79. Moresi V, Marroncelli N, Coletti D, Adamo S. Regulation of skeletal muscle development and homeostasis by gene imprinting, histone acetylation and microRNA. Biochimica et Biophysica Acta, 2015, 1849(3): 309-316 